\title{
Age-Related Changes in Nuclear Factor Erythroid 2-Related Factor 2 and Reactive Oxygen Species and Mitochondrial Structure in the Tongues of Fischer 344 Rats
}

\author{
Min-Kwan Baek $\cdot$ Hyon Lee ${ }^{2} \cdot$ Kyung-OK Kim ${ }^{3} \cdot$ Hyun-Jin Kwon ${ }^{3} \cdot$ Myung-Hee Chung ${ }^{4} \cdot$ Hyoung-Min Park ${ }^{1}$ \\ Joo-Hyun Woo ${ }^{1} \cdot$ Dong-Young Kim ${ }^{1}$ \\ Departments of ${ }^{1}$ Otolaryngology Head and Neck Surgery, ${ }^{2}$ Neurology, and ${ }^{3}$ Medical Research Institute, Gachon University Gil Hospital, Incheon; \\ ${ }^{4}$ Lee Gil Ya Cancer and Diabetes Institute, Gachon University, Incheon, Korea
}

Objectives. Previously the authors reported age-related changes in the activities of anti-oxidative enzyme activities and protein expressions in the tongues of rats. Because more information is required about relations between aging and oxidative stress and anti-oxidative enzyme efficiency, the authors investigated differences between the expression of master regulator of anti-oxidative enzymes (nuclear factor erythroid 2-related factor 2 [Nrf2]), levels of reactive oxygen species (ROS), and mitochondrial structures in the tongues of young and aged Fischer 344 rats.

Methods. Age-dependent changes in Nrf2 protein and ROS were determined by Western blotting and using chemical kits, respectively. Tongue specimens were examined by electron microscopy. The study was conducted using rats aged 7 months (young, $\mathrm{n}=8$ ) or 22 months (old, $\mathrm{n}=8$ ).

Results. Nrf2 protein levels in the tongues of aged rats were lower than in young rats. ROS levels were higher in older rats and mitochondrial structural deficits were observed their tongues. Three young rats showed moderate mitochondrial degeneration, whereas profound degeneration with mitochondrial cristae disruption, swelling, rupture, or intramitochondrial vacuole formation was observed in all 8 old rats. Notably, mitochondrial rupture was observed in 5 old rats.

Conclusion. Antioxidant defense systems of old rats were compromised by Nrf2 deficiency, which could lead to the deleterious accumulation and release of ROS and probably mitochondrial structural deficits in aged tongue tissues.

Keywords. Tongue; Aging; NF-E2-Related Factor 2; Reactive Oxygen Species; Mitochondria

\section{INTRODUCTION}

Aging is an unavoidable fact of life. Aging increases the vulnerability of cells to environmental insults, which leads to progres-

\footnotetext{
- Received August 18, 2016

Revised November 13, 2016

Accepted December 1, 2016

- Corresponding author: Dong-Young Kim

Department of Otolaryngology Head and Neck Surgery, Gachon University

Gil Hospital, 21 Namdong-daero 774beon-gil, Namdong-gu, Incheon

21565, Korea

Tel: +82-32-460-3762, Fax: +82-32-467-9044

E-mail: hndyk@gilhospital.com

*The first two authors contributed equally to this study and share first authorship.
}

sive, global impairment in tissue function, and increases the risks of disease and death [1]. Antioxidant enzymes, such as, glutathione peroxidase (GPX), catalase (CAT), and manganese superoxide dismutase (Mn-SOD), protect against the harmful effects of reactive oxygen species (ROS) by reducing them to hydrogen peroxide $\left(\mathrm{H}_{2} \mathrm{O}_{2}\right)$ and finally to water [2]. Aerodigestive organs are particularly susceptible to ROS damage, because they are continuously exposed to atmospheric oxygen [3]. However, only a few studies have addressed the subject of ROS-induced insults in tongues [4].

In a previous study, we showed the activities of total SOD, $\mathrm{GPx}$, and $\mathrm{Cu} / \mathrm{Zn}-\mathrm{SOD}$ were lower in the tongues of aged rats than in those of young rats, and that their protein expressions were down-regulated in old rats [5]. On the other hand, al-

Copyright (C) 2017 by Korean Society of Otorhinolaryngology-Head and Neck Surgery.

This is an open-access article distributed under the terms of the Creative Commons Attribution Non-Commercial License (http://creativecommons.org/licenses/by-nc/4.0)

which permits unrestricted non-commercial use, distribution, and reproduction in any medium, provided the original work is properly cited. 
though the protein expressions of Mn-SOD and CAT were lower in old rats, their enzyme activities were not [5]. Aging-associated impaired mitochondrial biogenesis has been associated with reduced levels and activities of nuclear factor erythroid 2-related factor 2 (Nrf2) [6]. In particular, the electron transport chain (ETC) in the inner membrane of mitochondria generates most ROS as a byproduct of the respiratory chain. Thus, mitochondria are exposed to the greatest amount of oxidative stress [7], which is defined as an imbalance between ROS production and antioxidant response [8].

The aim of this study was to document tongue Nrf2, ROS, and mitochondrial morphologic age-associated damage profiles.

\section{MATERIALS AND METHODS}

\section{Experimental animals}

Fischer 344 rats aged 7 months (young rats, $n=8$ ) and 22 months (old rats, $n=8$ ) were obtained from the Aging Tissue Bank (ATB) of Busan National University. Animals were anesthetized with carbon dioxide, and tongues were quickly removed. Epithelium and intrinsic muscle were extracted from the apex and body of tongues (anterior two thirds), excluding the tongue root. One portion of tongue tissue was sliced into $<1$ $\mathrm{mm}^{3}$ fragments, which were then placed in glutaraldehyde $(2.5 \%)$ for observation under a transmission electron microscope. Remaining tissue was minced on ice until homogenous, placed in liquid nitrogen, and stored at $-80^{\circ} \mathrm{C}$ until required.The Institutional Animal Care and Use Committee of Gachon University Gil Medical Center (Korea) approved all animal procedures (LCDI-2015-0023).

\section{Western immunoblotting (Nrf2)}

Tongues were homogenized $(10 \%, \mathrm{w} / \mathrm{v})$ at $4^{\circ} \mathrm{C}$ in a lysis buffer (Roche Diagnostics GmbH, Penzberg, Germany), which contained $150 \mathrm{mM} \mathrm{NaCl}, 1 \%$ Triton X-100 (Sigma-Aldrich, St. Louis, MO, USA), $20 \mathrm{mM}$ Tris-Cl (pH 7.4), 2 mM ethylene glycol-bis(2-aminoethylether)-N,N,N', $\mathrm{N}^{\prime}$-tetraacetic acid (EGTA) and protease inhibitors. Homogenates were centrifuged

\section{H I I G H L I I G}

- Nuclear factor erythroid 2-related factor 2 (Nrf2) protein levels were lower in the tongues of old rats than in those of young rats $(P<0.05)$.

- Reactive oxygen species (ROS) levels were higher in the tongues of older rats $(P<0.05)$.

- Mitochondrial structural deficits were observed in the tongues of older rats $(P<0.001)$.

- Antioxidants were compromised by Nrf2 deficiency, which can cause ROS accumulation and mitochondrial destruction. at $4^{\circ} \mathrm{C}$ for 10 minutes at $1,000 \times \mathrm{g}$, and nuclear fragments and tissue debris were removed. Proteins $(50 \mu \mathrm{g})$ were separated by 15\% SDS-PAGE (ProtoGel, National Diagnostics, Atlanta, GA, USA) and then transferred to nitrocellulose membranes, which were blocked at room temperature in $5 \%$ nonfat skim milk in phosphate-buffered saline (PBS) for 1 hour, and incubated overnight with primary antibodies for Nrf2 and GAPDH (Santa Cruz Biotechnology, Santa Cruz, CA, USA). After washing membranes three times in a Tris-buffered saline solution containing Tris-buffered saline solution containing $0.1 \%$ Tween 20 (TBST), they were incubated for 30 minutes with secondary antibodies (horseradish peroxidase-conjugated anti-rabbit IgGs [1:5,000]). After a final wash with TBST, an ECL detection system (Bio-Rad Laboratories Inc., Hercules, CA, USA) was used to visualize blots.

\section{Intracellular ROS assay}

ROS levels were determined using the OxiSelect In Vitro ROS/ RNS Assay Kit (Cell Biolabs Inc., San Diego, CA, USA). This method uses 2',7'-dichlorodihydrofluorescein DiOxyQ (DCFHDiOxyQ; a fluorogenic probe), which is deacetylated in cytosol to the non-fluorescent 2',7'-dichlorodihydrofluorescein (DCFH). DCFH rapidly reacts with ROS and reactive nitrogen species (RNS) (predominantly $\mathrm{NO}$ and $\mathrm{ONOO}^{-}$) to form the fluorescent product 2',7'-dichlorodihydrofluorescein (DCF). Thus, the total amount of ROS and RNS present is proportional to the intensity of DCF fluorescence $\left(\lambda_{\mathrm{ex}}=480 \mathrm{~nm}, \lambda_{\mathrm{em}}=530 \mathrm{~nm}\right)$. Briefly, cells $\left(1 \times 10^{6}\right.$ cells $\left./ \mathrm{mL}\right)$ were collected by centrifugation $(1,000 \times \mathrm{g}, 10$ minutes) and lysed using $300 \mu \mathrm{L}$ of $0.1 \%$ sodium dodecyl sulfate (SDS; dissolved in PBS) on ice for 30 minutes. The lysates were adjusted to equal protein concentrations (range, 1 to 10 $\mathrm{mg} / \mathrm{mL}$ ) using PBS. Protein concentrations were analyzed using the Bradford assay, and ROS/RNS levels were assayed using the above described OxiSelect kit using a calibration curve obtained using standard solutions of DCF in PBS. Absorbances were measured on a Tecan Infinite F200 PRO microplate reader (Tecan Austria GmbH, Mannedorf, Austria).

\section{Mitochondrial structures}

Samples of tongue tissues were cut into $<1 \mathrm{~mm}^{3}$ pieces, placed in glutaraldehyde $(2.5 \%)$ for 2 hours and then treated with osmium tetroxide (1\%) for 1 hour. Sections were stained with uranyl acetate 1\% (16 hours), dehydrated using an ethanol series, and embedded in Epon resin. Reynold's stain was used to stain Ultra-thin sections (600-900 A). A JEOL transmission electron microscope (JEOL Ltd, Tokyo, Japan) was used for the analysis.

Two sections per sample were processed for ultrastructural analysis by two observers. Mitochondrial degeneration in epithelial cells was assessed by awarding one point to each of the following: (1) mitochondrial cristae disruption; (2) intramitochondrial vacuole formation; (3) mitochondrial swelling; and (4) mitochondrial rupture. No differences were found between the 


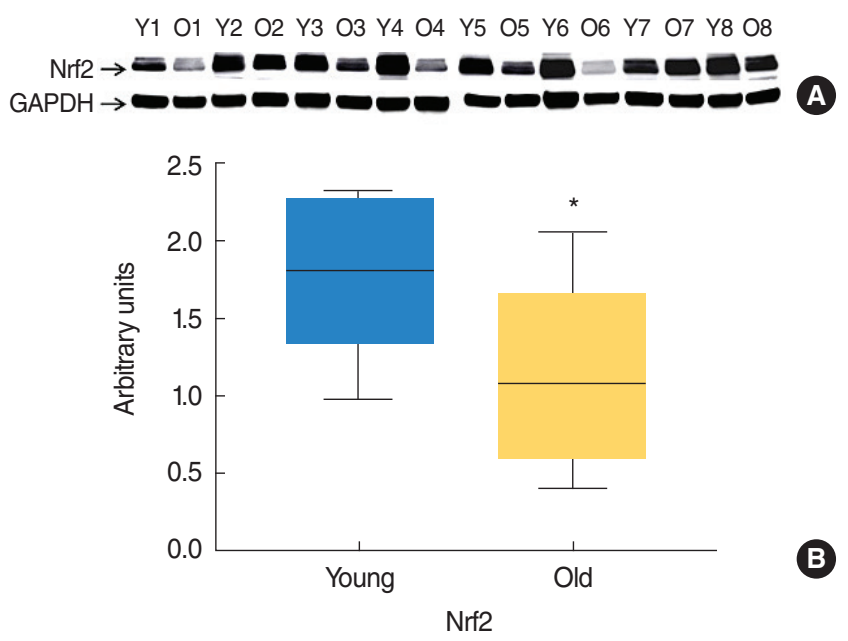

Fig. 1. Western blot analysis of nuclear factor erythroid 2-related factor 2 (Nrf2) protein expressions in the tongues of young (7 months) rats $(n=8)$ and old $(22$ months) rats $(n=8)$. Protein $(50 \mu \mathrm{g})$ lysates from tongue homogenates of young and old rats were resolved by SDS-PAGE (National Diagnostics) and probed with Nrf2 antibodies. Glyceraldehyde 3-phosphate dehydrogenase (GAPDH) was used as a loading control. (A) Western blot image of tongue samples. (B) Relative Nrf2 protein expressions were normalizing versus GAPDH. Results are expressed as median values. ${ }^{\star} P<0.05$ vs. young rats by the Mann-Whitney U-test.

assessments made by the two observers. Samples were classified as exhibiting normal, moderate, or profound degeneration based on the number of pathologic alterations observed. 'Normal' was defined as the presence of one or no pathologic alterations; moderate degeneration as two alterations, and profound degeneration as three of four alterations.

\section{Statistical analysis}

Significances of differences were determined using the MannWhitney $U$-test or Fisher exact test. $P$-values $<0.05$ were considered statistically significant. The statistical analysis was conducted using IBM SPSS ver. 20.0 (IBM Co.,Armonk, NY, USA).

\section{RESULTS}

Nrf2

Western blotting was used to assess Nrf2 protein levels in tongue tissues. Band densities were normalized versus GAPDH (the internal control). Relative expression levels of Nrf2 in the tongue tissues of 7- and 22-month-old rats are shown in Fig. 1. Notably, Nrf2 protein levels significantly decreased with age $(P=0.021)$.

\section{ROS}

ROS levels were significantly higher in the tongue tissues of old rats $(P=0.001$; old rats: $7,133 \pm 345$ and young rats: $5,247 \pm$ $418 \mathrm{nmol} \mathrm{DCF} / \mathrm{mg}$ tissue) (Fig. 2).

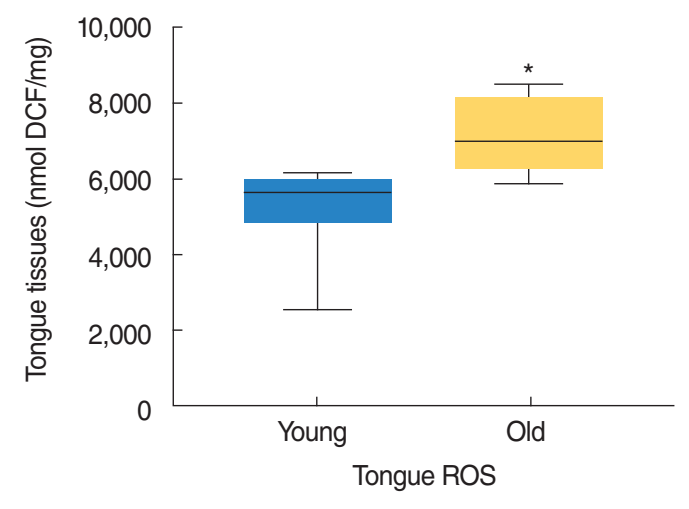

Fig. 2. Reactive oxygen species (ROS) levels in the tongue tissues of young ( 7 months) and old (22 months) ( $n=8$ animals/group). Results are expressed as median values, ${ }^{*} P<0.05$ vs. young rats by the Mann-Whitney U-test. DCF, 2',7'-dichlorodihydrofluorescein.

Table 1. Transmission electron microscopic findings of mitochondria in rat tongue samples and grades of mitochondrial degeneration in young and old animals

\begin{tabular}{|c|c|c|c|}
\hline & $\begin{array}{l}\text { Young } \\
\text { group } \\
(n=8)\end{array}$ & $\begin{array}{l}\text { Old } \\
\text { group } \\
(n=8)\end{array}$ & $P$-value \\
\hline \multicolumn{4}{|l|}{ Assessment points } \\
\hline Mitochondrial cristae disruption & $3(37.5)$ & $8(100)$ & 0.026 \\
\hline Intramitochondrial vacuole formation & $2(25.0)$ & $8(100)$ & 0.007 \\
\hline Mitochondrial swelling & $3(37.5)$ & $8(100)$ & 0.026 \\
\hline Mitochondrial rupture & 0 & $5(62.5)$ & 0.026 \\
\hline Grade of degenerationa) & & & $<0.001$ \\
\hline Normal appearance ${ }^{b)}$ & 5 & 0 & \\
\hline Moderate degenerationc) & 3 & 0 & \\
\hline Profound degeneration ${ }^{\mathrm{d})}$ & 0 & 8 & \\
\hline
\end{tabular}

Values are presented as number (\%).

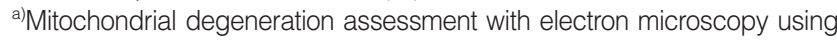

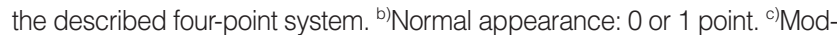
erate degeneration: 2 points. ${ }^{\text {d) }}$ Profound degeneration: $>2$ points.

\section{Ultrastructural analysis}

Mitochondrial alterations were present in 3 of the 8 young rats and in all 8 old rats $(n=8)$ (Table 1). These 3 young rats showed moderate degeneration with partial mitochondrial cristae disruption, mitochondrial swelling, or intramitochondrial vacuole formation (Fig. 3). Profound degeneration with mitochondrial cristae disruption, swelling, rupture, or intramitochondrial vacuole formation was observed in all 8 old rats (Fig. 4). Notably, mitochondrial rupture was observed in 5 old rats.

\section{DISCUSSION}

Nrf2 is a redox-sensitive transcription factor that functions in antioxidant defense, and typically is activated by xenobiotics [9], and may be involved in the pathogenesis of cell and tissue damage. In particular, Nrf2 plays a crucial role in protecting cells by 

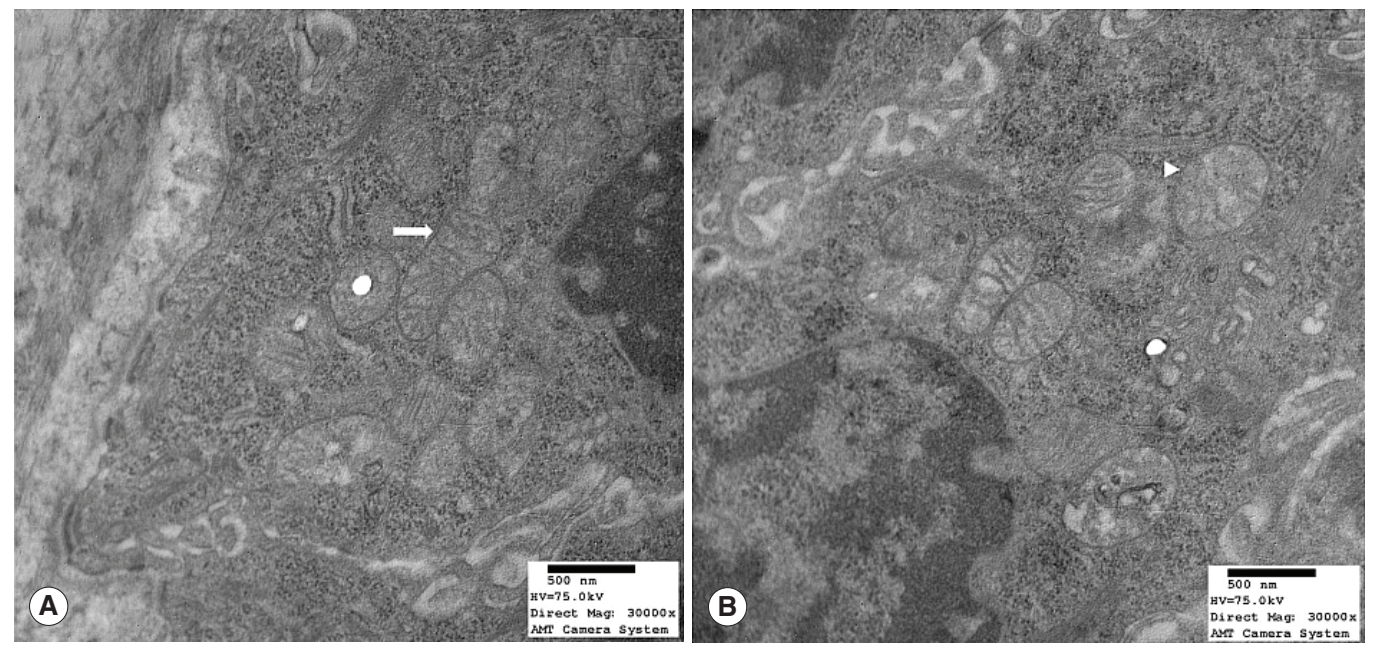

Fig. 3. Transmission electron microscopy image of mitochondrial structures in young ( 7 months) rats (Reynold's stain, $\times 30,000)$. (A) Mitochondria were normal and mitochondrial cristae were well preserved (arrow). (B) However, some mitochondrial cristae disruption and swelling (arrowhead) were observed.
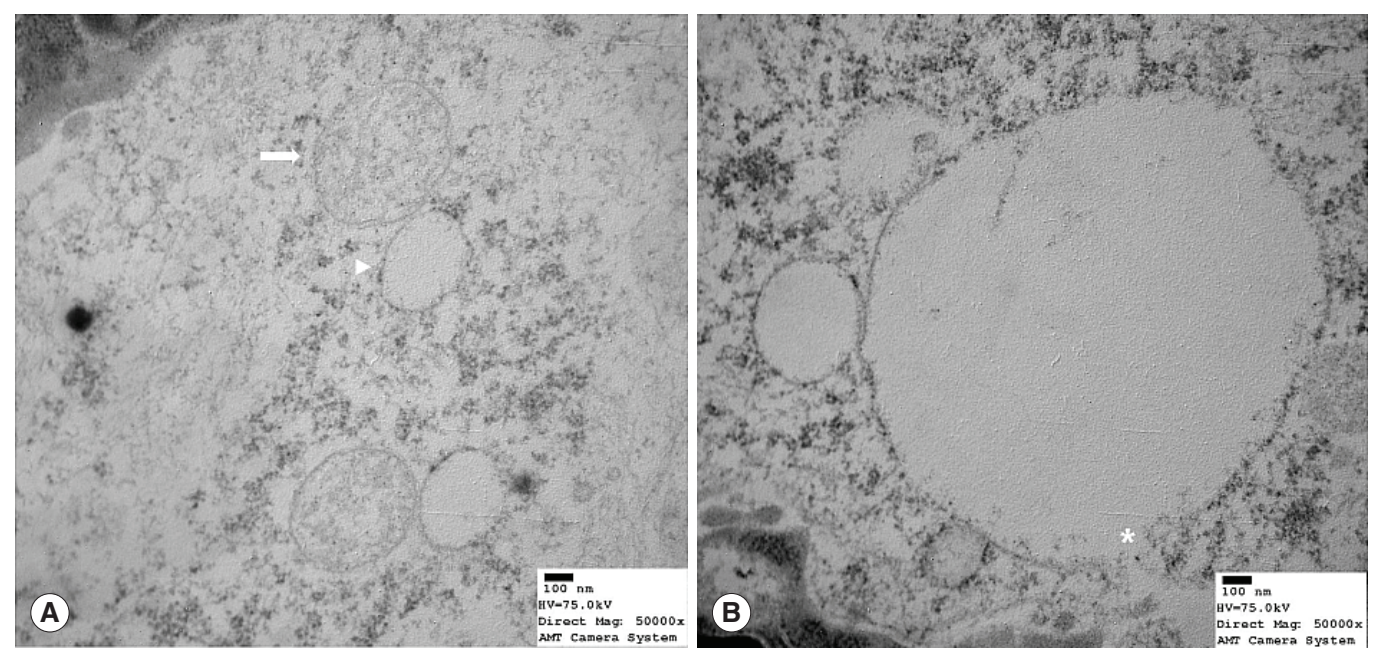

Fig. 4. Transmission electron microscope image showing mitochondrial structure in old (22 months) rats (Reynold's stain, $\times 50,000)$. (A) Profound degeneration was evidence by mitochondrial cristae loss, swelling (arrow), and vacuole formation (arrowhead). (B) Mitochondria showed cristae loss, vacuole formation, and membranous rupture (asterisk).

inducing the expressions of many cytoprotective genes following exposure to electrophilic or oxidative stress [10]. Keap1 is essential for Nrf2 activity regulation [11]. Normally, Nrf2 interacts with Keap1 in cytoplasm, and this interaction causes Nrf2 retention in cytoplasm and renders it liable to ubiquitination and proteasome degradation. However, Keap1 is inactivated when cells are exposed to ROS or electrophiles. This inactivation disrupts the Keap1/Nrf2 interaction and causes the release of Nrf2, which then translocate to the nucleus. In the nucleus Nrf2 activates the transcriptions of many genes that encode for enzymes and proteins that have detoxifying and antioxidant effects. In particular, the genes targeted by Nrf2 play roles in the synthesis of glutathione, ROS elimination, metabolism of xenobiotics, and protection against xenobiotic and oxidative stresses [12]. Ohkoshi et al. [13] reported Nrf2-knockout mice had a greater susceptibility to 4-nitroquinoline-1-oxide-induced carcinogenesis of the tongue and esophagus than wild-type mice.

In the present study, Western blotting showed that Nrf2 expression was suppressed in the tongue tissues of aged rats, which is in-line with our previous observation that antioxidant enzyme levels were diminished in aged rat tongues [5]. Smith et al. [14] focused on miRNA-146a, the expression of which leads to ageassociated reductions in Nrf2 expression. In addition, miRNA146a has been shown to block infections and treat inflammation, and functionally its effects are not diminished by aging. However, in aged rat's liver, it has been reported that the translocation 
of Nrf2 was reduced by $55 \%$. Additional studies are needed to measure miRNA-146a levels in tongues and to determine the influence of the interaction between miRNA-146a and Nrf2 on age-related degenerative changes observed in mitochondria.

Bone growth in rats tapers at 7 months, whereas human growth plates close in the 20 's. Thus, in the present study, we defined a 7-month-old rat to be equivalent to a 20 -year-old human. Female rats reach reproductive senescence at 15 to 25 months of age (average, 20 months) while in humans the average menopausal age is 51 years, which indicates a 22 -month-old rat is equivalent to a 55-60-year-old human (adult phase: 11.8 rat days $=1$ human year) [15]. Accordingly, in the present study, we defined 7- and 22-month-old rats as young and old rats, respectively.

Cell differentiation and proliferation can be promoted by a moderate increase in ROS level [16], whereas extreme increases can cause irreversible damage to bio-macromolecules and lead to apoptosis and cell death [17]. Therefore, the preservation of ROS balance is crucial for cellular health and survival. Furthermore, moderate ROS enhancement has been linked to cancer growth and the disruption of redox homeostasis [17]. Harris et al. [18] reported that normal epithelial cells exposed long-term to low amounts of exogenous oxidants became resistant to subsequent oxidative insults, which suggests cells are able to adapt and survive in the presence of oxidative stress, and that surviving cells have acquired mechanisms to counteract ROS toxicity and/or promote cell-survival pathways [16]. Oxidative damage by oxygen-derived free radicals has also been reported to be a causative factor of high mitochondrial DNA mutation rates in aged tissues [19]. Further studies are needed to assess whether aged tongue tissues are prone to certain mitochondrial DNA mutations and to identify the factors that contribute to such mutations. In the present study, ROS were found to be up-regulated in an aged rat tongues, and based on our previous finding that antioxidant enzyme levels were reduced in aged rat tongues [5], we suggest the up-regulation of ROS was possibly caused by diminished antioxidant enzyme levels.

Antioxidant enzymes importantly protect the cells of aerobic organs from oxidative stress. In particular, SOD's, such as, mitochondrial $\mathrm{Mn}-\mathrm{SOD}$ and cytosolic $\mathrm{Cu} / \mathrm{Zn}-\mathrm{SOD}$ reduce the highly toxic radical $\mathrm{O}_{2}^{-}$to less harmful $\mathrm{H}_{2} \mathrm{O}_{2}$, which is further reduced by CAT and GPx into water. However, if the levels of these antioxidant enzymes are diminished, ROS cannot be removed and its accumulation challenges nearby mitochondria [20]. In a previous study, our protein level and enzyme activity results indicated reduced antioxidant ability in the tongue tissues of older rats, despite similar enzyme Mn-SOD and CAT activities in old and young animals [5]. In another study, diminished glutathione (GSH) levels and elevated oxidized glutathione (GSSG) levels in the cardiac tissue of aged rats, caused CAT activity to increase as a compensatory mechanism to convert $\mathrm{H}_{2} \mathrm{O}_{2}$ to water [21]. We surmise this increase in CAT activity was higher in aged rats because metabolically active tissues are susceptible to aging-induced glutathione (GSH) depletion and redox state perturbations.

Mitochondria are known to be involved in the etiologies and pathogeneses of a variety of diseases and in the aging process $[22,23]$. Mitochondrial morphology may also change during aging in skeletal muscle, that is, some mitochondria become nonfunctional, depolarized, or enlarged [24]. Furthermore, as compared with young animals, which typically have elongated mitochondria, the mitochondria found in the skeletal muscle of aged animals are more rounded [25], which suggests the fusing ability of mitochondria may be compromised [2]. Aged mitochondria also become significantly swollen due to increased calcium retention [26]. In addition, in a previous study, deficient cristae formation was found to lead to the homogenization of constituents in mitochondrial compartments by transmission electron microscopy [27].

Degenerative changes in aged rat tongues have rarely have described in the literature. Barlagiannis et al. [28] reported that by 5 months, rat tongues already began to show evidence of degeneration, that is, moderate disruption of inter-epithelial junctions and lipid droplet formation, which concurs with our observations. Three 7-month-old rats exhibited moderate mitochondrial degeneration, whereas at 12 and 18 months, rat tongues showed profound degeneration, including edema of inter-epithelia, swelling of mitochondrial, lipid droplet formation, abnormal nuclear morphology, and cristae disruption. Takahashi [29] reported epithelium, propria mucosae, and muscle layers at the tongue apex became thin with aging, and that boundaries between these layers became unclear, which suggests some relationship between degenerative changes and motion dysfunction, pain, and paresthesia of the tongue. Choi et al. [30] reported age-related functional and structural changes of salivary glands, that is, acinar cell atrophy, cytoplasmic vacuolization, lymphocyte infiltration, smaller mucin component, and more periductal fibrosis in histomorphometric examinations of 90 -week-old mice. Additional studies are needed to determine whether the tongue undergoes similar age-related structural changes, and to characterize changes at the mitochondrial level in major head and neck organs.

This study expands understanding of the mechanism responsible for aging of the tongue. In particular, in old rats, attenuation of the antioxidant defense system, probably because of Nrf2 deficiency, might have increased the release and accumulation of ROS and caused mitochondrial structural deficits in aging tongue tissues.

\section{CONFLICT OF INTEREST}

No potential conflict of interest relevant to this article was reported. 


\section{ACKNOWLEDGMENTS}

The authors thank the Institute of Aging Tissue Bank (ATB) in Busan National University for providing animal tissues. This work was supported financially by the Gachon University Gil Medical Center Research Foundation.

\section{REFERENCES}

1. Kirkwood TB. Understanding the odd science of aging. Cell. 2005 Feb;120(4):437-47.

2. Huang JH, Hood DA. Age-associated mitochondrial dysfunction in skeletal muscle: contributing factors and suggestions for long-term interventions. IUBMB Life. 2009 Mar;61(3):201-14.

3. Geokas MC, Conteas CN, Majumdar AP. The aging gastrointestinal tract, liver, and pancreas. Clin Geriatr Med. 1985 Feb;1(1):177-205.

4. Vinokur V, Grinberg L, Berenshtein E, Gross M, Moskovitz J, Reznick AZ, et al. Methionine-centered redox cycle in organs of the aero-digestive tract of young and old rats. Biogerontology. 2009 Feb; 10(1):43-52.

5. Baek MK, Kim KO, Kwon HJ, Kim YW, Woo JH, Kim DY. Age-related changes in antioxidative enzyme capacity in tongue of fischer 344 rats. Clin Exp Otorhinolaryngol. 2016 Dec;9(4):352-7.

6. Sahin E, Colla S, Liesa M, Moslehi J, Muller FL, Guo M, et al.Telomere dysfunction induces metabolic and mitochondrial compromise. Nature. 2011 Feb;470(7334):359-65.

7. Meng Q, Wong YT, Chen J, Ruan R. Age-related changes in mitochondrial function and antioxidative enzyme activity in fischer 344 rats. Mech Ageing Dev. 2007 Mar;128(3):286-92.

8. Wu D, Cederbaum AI. Alcohol, oxidative stress, and free radical damage. Alcohol Res Health. 2003;27(4):277-84.

9. Motohashi H, Yamamoto M. Nrf2-Keap1 defines a physiologically important stress response mechanism. Trends Mol Med. 2004 Nov;10(11):549-57.

10. Itoh K, Chiba T, Takahashi S, Ishii T, Igarashi K, Katoh Y, et al. An $\mathrm{Nrf} 2 /$ small Maf heterodimer mediates the induction of phase II detoxifying enzyme genes through antioxidant response elements. Biochem Biophys Res Commun. 1997 Jul;236(2):313-22.

11. Itoh K, Wakabayashi N, Katoh Y, Ishii T, Igarashi K, Engel JD, et al. Keap1 represses nuclear activation of antioxidant responsive elements by Nrf2 through binding to the amino-terminal Neh2 domain. Genes Dev. 1999 Jan;13(1):76-86.

12. Moinova HR, Mulcahy RT. Up-regulation of the human gamma-glutamylcysteine synthetase regulatory subunit gene involves binding of Nrf-2 to an electrophile responsive element. Biochem Biophys Res Commun. 1999 Aug;261(3):661-8.

13. Ohkoshi A, Suzuki T, Ono M, Kobayashi T, Yamamoto M. Roles of Keap1-Nrf2 system in upper aerodigestive tract carcinogenesis. Cancer Prev Res (Phila). 2013 Feb;6(2):149-59.
14. Smith EJ, Shay KP, Thomas NO, Butler JA, Finlay LF, Hagen TM. Age-related loss of hepatic Nrf2 protein homeostasis: potential role for heightened expression of miR-146a. Free Radic Biol Med. 2015 Dec;89:1184-91.

15. Quinn R. Comparing rat's to human's age: how old is my rat in people years? Nutrition. 2005 Jun;21(6):775-7.

16. Barrera G. Oxidative stress and lipid peroxidation products in cancer progression and therapy. ISRN Oncol. 2012;2012:137289.

17. Trachootham D, Alexandre J, Huang P. Targeting cancer cells by ROS-mediated mechanisms: a radical therapeutic approach? Nat Rev Drug Discov. 2009 Jul;8(7):579-91.

18. Harris IS, Blaser H, Moreno J,Treloar AE, Gorrini C, Sasaki M, et al. PTPN12 promotes resistance to oxidative stress and supports tumorigenesis by regulating FOXO signaling. Oncogene. 2014 Feb;33 (8):1047-54.

19. Sohal RS, Ku HH, Agarwal S. Biochemical correlates of longevity in two closely related rodent species. Biochem Biophys Res Commun. 1993 Oct;196(1):7-11.

20. Ceballos-Picot I, Nicole A, Clement M, Bourre JM, Sinet PM. Agerelated changes in antioxidant enzymes and lipid peroxidation in brains of control and transgenic mice overexpressing copper-zinc superoxide dismutase. Mutat Res. 1992;275(3-6):281-93.

21. Kumaran S, Savitha S, Anusuya Devi M, Panneerselvam C. L-carnitine and DL-alpha-lipoic acid reverse the age-related deficit in glutathione redox state in skeletal muscle and heart tissues. Mech Ageing Dev. 2004 Jul;125(7):507-12.

22. Beal MF. Does impairment of energy metabolism result in excitotoxic neuronal death in neurodegenerative illnesses? Ann Neurol. 1992 Feb;31(2):119-30.

23. Diplock AT. Antioxidants and disease prevention. Mol Aspects Med. 1994;15(4):293-376.

24. Terman A, Gustafsson B, Brunk UT. The lysosomal-mitochondrial axis theory of postmitotic aging and cell death. Chem Biol Interact. 2006 Oct;163(1-2):29-37.

25. de Cavanagh EM, Piotrkowski B, Basso N, Stella I, Inserra F, Ferder $\mathrm{L}$, et al. Enalapril and losartan attenuate mitochondrial dysfunction in aged rats. FASEB J. 2003 Jun;17(9):1096-8.

26. Kumaran S, Panneerselvam KS, Shila S, Sivarajan K, Panneerselvam C. Age-associated deficit of mitochondrial oxidative phosphorylation in skeletal muscle: role of carnitine and lipoic acid. Mol Cell Biochem. 2005 Dec;280(1-2):83-9.

27. Beregi E, Regius O, HuttlT, Gobl Z. Age-related changes in the skeletal muscle cells. Z Gerontol. 1988 Mar-Apr;21(2):83-6.

28. Barlagiannis D, Dietrich E, Papaliagkas V, Makri S, Toskas A, Papamitsou T. Ultrastructural aspects of the effects of L-carnitine administration on epithelial cells in the aging rat tongue. Hippokratia. 2014 Jan;18(1):32-6.

29. Takahashi T. Structual changes of the apex region of the tongue in the elderly. Kokubyo Gakkai Zasshi. 2008 Jun;75(2):93-105.

30. Choi JS, Park IS, Kim SK, Lim JY, Kim YM. Analysis of age-related changes in the functional morphologies of salivary glands in mice. Arch Oral Biol. 2013 Nov;58(11):1635-42. 